\section{ASIRTaining a Parkinson's disease target}

\section{By Lev Osherovich, Senior Writer}

A Tufts University team has compelling evidence that reducing sirtuin 2 activity could be beneficial in Parkinson's disease. ${ }^{1}$ The findings argue for developing brain-penetrating sirtuin 2 inhibitors.

Sirtuin 2 (SIRT2) is one of seven human sirtuins, a class of protein deacetylases with diverse roles in metabolic, inflammatory and autoimmune diseases. The best-studied member of the class is SIRT1, which first emerged in the 2000s when Harvard Medical School researchers showed that overexpressing or activating the target promoted disease resistance in multiple preclinical models of metabolic and neurological diseases. ${ }^{2}$

Based on those findings, Sirtris Pharmaceuticals Inc. was founded in 2004 to develop small molecule activators of sirtuins. The company was acquired by GlaxoSmithKline plc in 2008 for \$651 million.

Since then, sirtuin activators have encountered roadblocks. In 2010, GSK discontinued development of Sirtris' lead compound, SRT510, and trials of next-
"A genetic knockout is useful but not exactly what you'd expect with pharmacological inhibition due to compensatory regulation of other sirtuins."

- George Vlasuk, Sirtris Pharmaceuticals Inc. of SIRT2 was beneficial in fly, worm and cell culture models of $\mathrm{HD},{ }^{4}$ although those conclusions were challenged by a 2012 study from MIT and King's College London researchers showing no effect of Sirt2 deletion in a mouse model of HD. ${ }^{5}$

Earlier this year, a Harvard Medical School team showed that hitting SIRT2 with a relatively nonselective inhibitor ameliorated frontotemporal dementia in mice. ${ }^{6}$

Now, a team led by Gizem Donmez, assistant professor of neuroscience at the Tufts University School of Medicine and lead author of the previous AD study of SIRT1, has analyzed the role of Sirt2 in PD in mice.

Donmez tested the effect of deleting the enzyme in a widely used mouse model of PD in which the disease is induced by treatment with the neurotoxin 1-methyl-4-phenyl-1,2,3,6-tetrahydropyridine (MPTP).

Her team's conclusion is that inhibiting SIRT2 seems to be beneficial. "We have shown that MPTP causes death of neurons via apoptosis, and activation of this pathway is diminished by SIRT2 inhibition," said Donmez.

\section{Damage resistance}

Donmez's team chronically administered MPTP to young Sirt2 knockout mice and wild-type littermates and looked for evidence of damage in the substantia nigra, the brain region most affected by PD.

Brain slices from wild-type mice treated with MPTP had fewer dopaminergic neurons than those treated with saline. In contrast, Sirt2 knockout mice treated with MPTP had similar numbers of neurons.

The group then tested the effect of modulating SIRT2 levels on apoptosis, which is thought to be the main mechanism of dopaminergic cell death in PD. Upon MPTP treatment, cultured human neuroblastoma generation SIRT1 activators in a range of metabolic and inflammatory conditions have not advanced beyond Phase II testing.

SRT2104, the most advanced next-generation SIRT1 activator, completed Phase I/II trials for type 2 diabetes, but GSK said it is no longer pursuing that indication with the compound. SRT2104 remains in Phase IIa development for ulcerative colitis (UC).

Although efforts to activate sirtuins in peripheral diseases have thus far yielded little fruit, academic researchers have been building a case for targeting the family in neurodegenerative diseases.

In the nervous system, sirtuins are thought to activate prosurvival pathways in response to stressful conditions such as Alzheimer's disease (AD), Huntington's disease (HD) and PD, thus exerting a broadly neuroprotective effect.

Indeed, genetic evidence argues that activating SIRT1 would be helpful in AD. For example, researchers at the Massachusetts Institute of Technology reported in 2010 that genetic deletion of Sirt 1 exacerbated AD in mice. ${ }^{3}$

SIRT2 appears to play an opposing role to SIRT1 in neurodegenerative diseases. In 2010, another MIT team reported that deletion or inhibition cells expressing a small hairpin RNA construct that knocked down SIRT2 expression had lower levels of apoptotic markers than vectortreated cells.

Conversely, overexpression of SIRT2 increased apoptotic markers compared with normal expression of SIRT2.

Results were published in The Journal of Biological Chemistry and were not patented.

\section{Opposite effects}

Donmez said the findings add in vivo validation to prior in vitro evidence for targeting SIRT2 in PD, ${ }^{7}$ but further studies with other mouse models of PD are needed to really shore up the conclusion. Indeed, testing the effect of Sirt2 deletion in mice with various mutations associated with hereditary PD are her next steps.

The new findings also add to evidence for the opposing roles for SIRT1 and SIRT2 in preserving neurons from disease-associated damage. Although both proteins are thought to be activated by cellular stresses such as MPTP exposure, it is not clear why they should have opposite effects on cell survival. 


\section{"SIRT1 and SIRT2 may have a yin-yang relationship." \\ -Gizem Donmez, \\ Tufts University School of Medicine}

One possibility is that deletion or inactivation of SIRT2 could lead to activation of SIRT1. If so, it may still be desirable to activate SIRT1 to treat PD.

"SIRT1 and SIRT2 may have a yin-yang relationship," said Donmez.

Another scenario is that the two enzymes have truly unrelated functions.

"SIRT2 is very distinct from SIRT1," said George Vlasuk, CEO of GSK's Sirtris unit. "They have the same catalytic domain but the regulation and intracellular distribution of these enzymes is very different."

He noted that SIRT2 is located primarily in the cytoplasm, whereas SIRT1 is primarily in the nucleus.

Vlasuk would like to see the effect of Sirt2 deletion in functional tests of PD progression such as motor activity and gait.

In addition, both Vlasuk and Donmez cautioned that genetic deletion of sirtuins can have different effects than inhibiting the enzymes with small molecules.

"A genetic knockout is useful but not exactly what you'd expect with pharmacological inhibition due to compensatory regulation of other sirtuins," said Vlasuk. "What are needed are selective pharmacological inhibitors of SIRT2 that are brain penetrant."

In June, a Japanese academic team published in vitro studies of potent selective inhibitors of SIRT2 ${ }^{8}$ However, the brain-penetrating properties of these compounds have not yet been tested in mouse models of PD.

Siena Biotech S.p.A. has selisistat (EX-527), an inhibitor of SIRT1, in Phase II testing for HD.

Osherovich, L. SciBX 5(37); doi:10.1038/scibx.2012.972

Published online Sept. 20, 2012

REFERENCES

1. Lui, L. et al. J. Biol. Chem.; published online Aug. 16, 2012; doi:10.1074/jbc.C112.403048

Contact: Gizem Donmez, Tufts University School of Medicine, Boston, Mass. e-mail: gizem.donmez@tufts.edu

2. Herranz, D. \& Serrano, M. Nat. Rev. Cancer 10, 819-823 (2010)

3. Donmez, G. et al. Cell 142, 320-332 (2010)

4. Luthi-Carter, R. et al. Proc. Natl. Acad. Sci. USA 107, 7927-7932 (2010)

5. Bobrowska, A. et al. PloS ONE 7, e34805; published online April 12 , 2012; doi:10.1371/journal.pone.0034805

6. Spires-Jones, T.L. et al. Front. Pharmacol. 3, 42; published online March 12, 2012; doi:10.3389/fphar.2012.00042

7. Outeiro, T.F. et al. Science 317, 516-519 (2007)

8. Suzuki, T. et al. J. Med. Chem. 55, 5760-5773 (2012)

COMPANIES AND INSTITUTIONS MENTIONED

Harvard Medical School, Boston, Mass.

GlaxoSmithKline plc (LSE:GSK; NYSE:GSK), London, U.K.

King's College London, London, U.K.

Massachusetts Institute of Technology, Cambridge, Mass.

Siena Biotech S.p.A., Siena, Italy

Sirtris Pharmaceuticals Inc. (NASDAQ:SIRT), Cambridge, Mass.

Tufts University, Boston, Mass.

Tufts University School of Medicine, Boston, Mass. 